

\title{
Two-photon Imaging of Microglial Processes' Attraction Toward ATP or Serotonin in Acute Brain Slices
}

Fanny Etienne, Vincenzo Mastrolia, Luc Maroteaux, Jean-antoine Girault, Nicolas Gervasi, Anne Roumier

\section{- To cite this version:}

Fanny Etienne, Vincenzo Mastrolia, Luc Maroteaux, Jean-antoine Girault, Nicolas Gervasi, et al.. Two-photon Imaging of Microglial Processes' Attraction Toward ATP or Serotonin in Acute Brain Slices. Journal of visualized experiments : JoVE, 2019, 143, 10.3791/58788 . hal-02348070

\author{
HAL Id: hal-02348070 \\ https://hal.science/hal-02348070
}

Submitted on 5 Nov 2019

HAL is a multi-disciplinary open access archive for the deposit and dissemination of scientific research documents, whether they are published or not. The documents may come from teaching and research institutions in France or abroad, or from public or private research centers.
L'archive ouverte pluridisciplinaire HAL, est destinée au dépôt et à la diffusion de documents scientifiques de niveau recherche, publiés ou non, émanant des établissements d'enseignement et de recherche français ou étrangers, des laboratoires publics ou privés. 


\section{TITLE:}

2 Two-photon Imaging of Microglial Processes' Attraction Toward ATP or Serotonin in Acute Brain 3 Slices

AUTHORS \& AFFILIATIONS:

6 Fanny Etienne ${ }^{1,2,3}$, Vincenzo Mastrolia ${ }^{1,2,3}$, Luc Maroteaux ${ }^{1,2,3}$, Jean-Antoine Girault ${ }^{1,2,3}$, Nicolas 7 Gervasi ${ }^{1,2,3} *$, Anne Roumier ${ }^{1,2,3} *$

${ }^{1}$ Institut national de la santé et de la recherche médicale (INSERM), Institut du Fer-à-Moulin

* These authors contributed equally.

\section{Corresponding Authors:}

Anne Roumier (anne.roumier@inserm.fr)

Phone: +33 145876124

Nicolas Gervasi（nicolas.gervasi@inserm.fr)

Phone: +33145876153

\section{E-mail Addresses of the Co-authors:}

$\begin{array}{ll}\text { Fanny Etienne } & \text { (Fanny.etienne@inserm.fr) } \\ \text { Vincenzo Mastrolia } & \text { (Vincenzo.mastrolia@inserm.fr) } \\ \text { Luc Maroteaux } & \text { (Luc.maroteaux@inserm.fr) } \\ \text { Jean-Antoine Girault } & \text { (Jean-antoine.girault@inserm.fr) }\end{array}$

\section{KEYWORDS:}

Microglia, multi-photon microscopy, acute brain slices, chemoattraction, motility, morphology, serotonin, ATP, live imaging, time-lapse imaging

\section{SUMMARY:}

Microglia, the resident immune cells of the brain, respond quickly with morphological changes to modifications of their environment. This protocol describes how to use two-photon microscopy to study the attraction of microglial processes toward serotonin or ATP in acute brain slices of mice.

\section{ABSTRACT:} environment with their long processes and, upon disruption of homeostasis, undergo rapid morphological changes. For example, a laser lesion induces in a few minutes an oriented growth of microglial processes, also called "directional motility", toward the site of injury. A similar effect can be obtained by delivering locally ATP or serotonin (5-hydroxytryptamine $[5-\mathrm{HT}]$ ). In this 
article, we describe a protocol to induce a directional growth of microglial processes toward a local application of ATP or 5-HT in acute brain slices of young and adult mice and to image this attraction over time by multiphoton microscopy. A simple method of quantification with free and open-source image analysis software is proposed. A challenge that still characterizes acute brain slices is the limited time, decreasing with age, during which the cells remain in a physiological state. This protocol, thus, highlights some technical improvements (medium, air-liquid interface chamber, imaging chamber with a double perfusion) aimed at optimizing the viability of microglial cells over several hours, especially in slices from adult mice.

\section{INTRODUCTION:}

Microglial cells are the brain's resident macrophages and play a role in both physiological and pathological conditions ${ }^{1,2}$. They have a highly branched morphology and are constantly extending and retracting their processes ${ }^{3,4}$. This "scanning" behavior is believed to be related and necessary to the survey of their surroundings. The morphological plasticity of microglia is expressed in three modes. First, some compounds rapidly modulate microglial morphology: the addition of ATP ${ }^{5,6}$ or $\mathrm{NMDA}^{5,7}$ in the medium bathing acute brain slices increases the complexity of microglial ramifications, whereas norepinephrine decreases $i^{6}$. These effects either are directly mediated by microglial receptors (for ATP and norepinephrine) or require an ATP release from neurons (for NMDA). Second, the growth and retraction speed of microglial processes, called motility or "surveillance", can be affected by extracellular factors ${ }^{8}$, homeostasis disruptions ${ }^{9,10}$, or mutations ${ }^{9-11}$. Third, in addition to these isotropic changes of morphology and motility, microglia have the capacity to extend their processes directionally toward a pipette delivering ATP ${ }^{3,5,12-14}$, in culture, in acute brain slices or in vivo, or delivering $5-\mathrm{HT}$ in acute brain slices ${ }^{15}$. Such oriented growth of microglial processes, also called directional motility, was first described as a response to a local laser lesion ${ }^{3,4}$. Thus, physiologically, it may be related to the response to injury or required for targeting microglial processes toward synapses or brain regions requiring pruning during development ${ }^{15,16}$, or in physiological ${ }^{17-19}$ or pathological situations $s^{9,18-20}$ in adulthood. The three types of morphological changes rely on different intracellular mechanisms ${ }^{11,13,20}$, and one given compound does not necessarily modulate all of them (e.g., NMDA, which acts indirectly on microglia, has an effect on morphology but does not induce directional motility ${ }^{5,7}$ ). Therefore, when aiming to characterize the effect of a compound, a mutation or a pathology on microglia, it is important to characterize the three components of their morphological plasticity. Here, we describe a method to study the directional growth of microglial processes toward a local source of compound, which is, here, ATP or 5-HT.

There are several models to study microglia processes' attraction: primary cultures in 3D environment ${ }^{6,18,19}$, acute brain slices ${ }^{6,13,15}$, and in vivo imaging ${ }^{3,13}$. The in vivo approach is the best to preserve the physiological state of microglia. However, intravital imaging of deep regions requires complex surgical procedures and, therefore, it is often limited to superficial cortical layers. The use of microglia primary culture is the easiest technique to test a large number of conditions with a limited number of animals. Nevertheless, it is impossible to obtain the same cell morphology as in vivo, and cells lose their physiological interactions with neurons and astrocytes. Acute brain slices represent a compromise between these two approaches. This model allows researchers to study brain structures which are otherwise difficult to reach and to 
image with high resolution in vivo, and to investigate slices from neonatal stages, whereas transcranial microscopy is mostly performed at adulthood. Finally, it makes it possible to observe in real-time the effects of local drug application, and to repeat experiments while using a limited number of animals. Nonetheless, an issue with acute brain slices is the limited time (a few hours) during which the cells remain alive, notably for slices from mice older than two weeks, and the potential change of microglia morphology over time ${ }^{21,22}$.

Here, we describe a protocol to prepare acute brain slices of young and adult $\mathrm{C} \times 3 \mathrm{cr} 1^{\mathrm{GFP} /+}$ mice up to two months old, with the preservation of microglia morphology and motility for several hours. We, then, describe how to use these slices to study the attraction of microglial processes toward compounds like ATP or 5-HT.

\section{PROTOCOL:}

All experiments were approved by the local ethical committee (Darwin Committee, agreements $\# 1170$ and \#10921).

\section{Preparation of Glass Micropipettes for the Local Application of Compounds}

1.1. Prepare pipettes from borosilicate thin-wall glass capillaries with an electrode puller. Adjust the parameters to obtain pipettes with a 4 - $5 \mu \mathrm{m}$ diameter at their extremity. Figure 2D shows one pipette in brightfield at low magnification.

\section{Solutions}

2.1. Ensure that only glassware that has been cleaned by an autoclave cycle, followed by rinsing $2 x-3 x$ with ultrapure water, will be used. Never use glassware that has been in contact with paraformaldehyde.

2.2. Prepare a $2 \mathrm{~mol} \cdot \mathrm{L}^{-1} \mathrm{CaCl}_{2}$ stock solution by dissolving $14.7 \mathrm{mg}$ of $\mathrm{CaCl}_{2} \cdot 2 \mathrm{H}_{2} \mathrm{O}$ in $50 \mathrm{~mL}$ of water of high purity (ultrapure water, resistance $18.2 \mathrm{M} \Omega$; the traces of metal in distilled water or tap water can lead to suboptimal slice quality due to pro-oxidative effects).

\subsubsection{Store this stock solution at room temperature for a maximum of one month.}

2.3. On the day of the experiment, prepare $1 \mathrm{~L}$ of choline-aCSF (artificial cerebrospinal fluid) solution, whose composition is $110 \mathrm{mmol} \cdot \mathrm{L}^{-1}$ choline $\mathrm{Cl}, 25 \mathrm{mmol} \cdot \mathrm{L}^{-1}$ glucose, $25 \mathrm{mmol} \cdot \mathrm{L}^{-1}$ $\mathrm{NaHCO}_{3}, 7 \mathrm{mmol} \cdot \mathrm{L}^{-1} \mathrm{MgCl}_{2}, 11.6 \mathrm{mmol} \cdot \mathrm{L}^{-1}$ ascorbic acid, $3.1 \mathrm{mmol} \cdot \mathrm{L}^{-1}$ sodium pyruvate, 2.5 $\mathrm{mmol} \cdot \mathrm{L}^{-1} \mathrm{KCl}, 1.25 \mathrm{mmol} \cdot \mathrm{L}^{-1} \mathrm{NaH}_{2} \mathrm{PO}_{4}$, and $0.5 \mathrm{mmol} \cdot \mathrm{L}^{-1} \mathrm{CaCl}_{2}, 0.5$.

2.3.1. To prepare this solution, add, in the following order, to a $1 \mathrm{~L}$ graduated flask: $0.186 \mathrm{~g}$ of $\mathrm{KCl}$, $0.195 \mathrm{~g}$ of $\mathrm{NaH}_{2} \mathrm{PO}_{4}, 2.04 \mathrm{~g}$ of acid ascorbic, $2.1 \mathrm{~g}$ of $\mathrm{NaHCO}_{3}$, and $4.5 \mathrm{~g}$ of glucose.

2.3.2. Fill about half of the final volume with ultrapure water and stir until complete dissolution. 
2.3.3. Add $0.34 \mathrm{~g}$ of sodium pyruvate and $15.36 \mathrm{~g}$ of choline $\mathrm{Cl}$.

NOTE: It is convenient to first dissolve the choline $\mathrm{Cl}$ with 5 to $10 \mathrm{~mL}$ of the solution prepared in step 2.3.2 before adding it to the whole solution.

2.3.4. Add $7 \mathrm{~mL}$ of $1 \mathrm{~mol} \cdot \mathrm{L}^{-1} \mathrm{MgCl}_{2}$ and $250 \mu \mathrm{L}$ of $2 \mathrm{~mol} \cdot \mathrm{L}^{-1} \mathrm{CaCl}_{2}$ (prepared in step 2.2) to the solution.

2.3.5. Fill the graduated flask up to $1 \mathrm{~L}$ with ultrapure water.

2.3.6. With a vapor pressure osmometer, check that the osmolarity is between 300 and $310 \mathrm{~m} \Omega$. If not, adjust it with glucose.

2.3.7. Check the $\mathrm{pH}$ after carbogenation (i.e., bubbling with "carbogen", a mix of $95 \% \mathrm{O}_{2} / 5 \% \mathrm{CO}_{2}$ ) and adjust it, if necessary, to $7.3-7.4$ with $10 \mathrm{M} \mathrm{NaOH}$.

2.3.8. Transfer the solution to a glass bottle for storage. Keep the bottle in the fridge until use (STEP 3.1).

NOTE: It is recommended to make a fresh solution on the day of the experiment. However, if necessary, choline-aCSF can be stored up to two days at $4{ }^{\circ} \mathrm{C}$.

2.4. On the day of the experiment, prepare $1 \mathrm{~L}$ of an aCSF solution, whose composition is 124 $\mathrm{mmol} \cdot \mathrm{L}^{-1} \mathrm{NaCl}, 26.2 \mathrm{mmol} \cdot \mathrm{L}^{-1} \mathrm{NaHCO}_{3}, 25 \mathrm{mmol} \cdot \mathrm{L}^{-1}$ glucose, $2.5 \mathrm{mmol} \cdot \mathrm{L}^{-1} \mathrm{KCl}, 2 \mathrm{mmol} \cdot \mathrm{L}^{-1} \mathrm{CaCl}_{2}, 1$ $\mathrm{mmol} \cdot \mathrm{L}^{-1} \mathrm{MgCl}$, and $1.25 \mathrm{mmol} \cdot \mathrm{L}^{-1} \mathrm{NaH}_{2} \mathrm{PO}_{4}$.

2.4.1. To prepare this solution, add, in the following order, to a graduated flask: $0.150 \mathrm{~g}$ of $\mathrm{NaH}_{2} \mathrm{PO}_{4}, 0.186 \mathrm{~g}$ of $\mathrm{KCl}, 2.2 \mathrm{~g}$ of $\mathrm{NaHCO}_{3}, 4.5 \mathrm{~g}$ of glucose, and $7.3 \mathrm{~g}$ of $\mathrm{NaCl}$. Bring the solution to a volume of $1 \mathrm{~L}$ with ultrapure water and stir it vigorously on a stir plate.

2.4.2. Add $1 \mathrm{~mL}$ of $1 \mathrm{~mol} \cdot \mathrm{L}^{-1} \mathrm{MgCl}_{2}$ and $1 \mathrm{~mL}$ of $2 \mathrm{~mol} \cdot \mathrm{L}^{-1} \mathrm{CaCl}_{2}$ to the solution and transfer the aCSF solution to a glass bottle for storage.

2.4.3. Check whether the osmolarity is $300-310 \mathrm{~m} \Omega \cdot \mathrm{L}^{-1}$ and, if not, adjust it with glucose.

2.4.4. Check the $\mathrm{pH}$ after carbogenation (i.e., bubbling with "carbogen") and adjust it, if necessary, to $7.3-7.4$ with $10 \mathrm{M} \mathrm{NaOH}$.

2.4.5. Transfer the solution to a glass bottle for storage. Keep the bottle in the fridge until use (step 3.1).

NOTE: It is recommended to make a fresh solution on the day of the experiment. However, an alternative is to prepare a $10 x$ stock solution containing $\mathrm{NaCl}, \mathrm{NaHCO}_{3}, \mathrm{KCl}$, and $\mathrm{NaH}_{2} \mathrm{PO}_{4}$ at $10 \mathrm{x}$ the final concentration, which can be stored for no more than one week at $4{ }^{\circ} \mathrm{C}$. Make the final 
aCSF on the day of the experiment by diluting the 10x stock solution with ultrapure water and adding the glucose, $\mathrm{CaCl}_{2}$, and $\mathrm{MgCl}_{2}$.

2.5. Prepare the drug solutions on the day of the experiment. Use the aCSF solution to bring them to the final concentrations which are, here, $500 \mu \mathrm{mol} \cdot \mathrm{L}^{-1}$ for ATP and $5 \mu \mathrm{mol} \cdot \mathrm{L}^{-1}$ for $5-\mathrm{HT}$.

NOTE: For ATP, a stock solution can be prepared (e.g., $50 \mathrm{mM}$ ATP in water), stored in aliquoted form at $-20^{\circ} \mathrm{C}$, and diluted with aCSF to the final concentration on the day of the experiment. In contrast, the 5-HT (serotonin- $\mathrm{HCl}$ ) solution must be prepared from powder on the day of the experiment, at $1 \mathrm{mg} \cdot \mathrm{mL}^{-1}$ in water, kept at $4{ }^{\circ} \mathrm{C}$ to avoid $5-\mathrm{HT}$ oxidation, and diluted in aCSF at the time of the experiment.

\section{Preparation of Acute Brain Slices}

\subsection{Preparation of the dissection area}

3.1.1. Prepare $70 \mathrm{~mL}$ of ice-cold choline-aCSF in an $80 \mathrm{~mL}$ beaker placed on ice, to be used for cardiac perfusion, rapid cooling down of the brain, and slicing. Prepare $150 \mathrm{~mL}$ of choline-aCSF in a $200 \mathrm{~mL}$ crystallizing dish, placed in a heated water bath maintained at $32^{\circ} \mathrm{C}$. Place a nylon mesh strainer in the crystallizing dish to retain the slices. This will be used to let the slices recover for 10 min just after slicing.

3.1.2. At least $30 \mathrm{~min}$ before starting the dissection (section 3.2), start bubbling these two solutions ( $70 \mathrm{~mL}$ of choline-aCSF on ice and $150 \mathrm{~mL}$ of choline-aCSF at $32^{\circ} \mathrm{C}$ ) with carbogen. Maintain constant carbogenation during the entire procedure.

3.1.3. Prepare the interface chamber device (Figure 1C), which will be used to keep slices until their use.

3.1.3.1. In a sealed food box ( $10 \times 10 \mathrm{~cm}$ or $10 \mathrm{~cm}$ in diameter, $8 \mathrm{~cm}$ in height), installed on a magnetic stirrer, place a $200 \mathrm{~mL}$ crystallizing dish with a bar magnet.

3.1.3.2. Add $200 \mathrm{~mL}$ of aCSF in this crystallizing dish and place the 3D-printed interface slice holder on top of it (the interface slice holder is composed of two perfectly fitting parts, with a polyamide mesh stretched between them, Figure 1A,B).

3.1.3.3. Remove excess volume from the crystallizing dish to keep only a thin film of solution covering the mesh of the interface slice holder. This will later create a fine rim of solution surrounding the slices (but without covering them).

3.1.3.4. Put a few millimeters of aCSF at the bottom of the food box and start bubbling it with carbogen (at first use, make a small hole in the sealed food box wall to make sure the tubing can enter the box). 
3.1.3.5. Close the sealed box while maintaining constant carbogenation. This will create a humidified $95 \% \mathrm{O}_{2} / 5 \% \mathrm{CO}_{2}$ rich environment in which the slices will be transferred after their recovery in choline-aCSF and maintained before they are imaged. This device is hereafter referred to as the "interface chamber" (Figure 1C).

\subsection{Brain dissection and slicing}

3.2.1. Anesthetize the mouse with an intraperitoneal injection of $50 \mathrm{mg} \cdot \mathrm{mL}^{-1}$ pentobarbital $(0.15$ $\mathrm{mL} / 20 \mathrm{~g}$ of mouse body weight), immobilize it, expose the heart, and perform a cardiac perfusion with $10 \mathrm{~mL}$ of ice-cold, carbogenated, choline-aCSF (see step 3.1.1), with a peristaltic pump. Observe the pallor of the liver as an indicator of a good perfusion. The perfusion lasts less than 5 $\min$.

3.2.2. Decapitate the mouse and cut the skin to expose the skull. With big scissors, apply two transversal cuts from the large foramen and one long sagittal cut and, using fine forceps, remove the skull plates.

3.2.3. Quickly and gently extract the brain (in less than $1 \mathrm{~min}$ ) and place it for $1 \mathrm{~min}$ in the $80 \mathrm{~mL}$ beaker containing the remaining $(\sim 60 \mathrm{~mL}$ ) ice-cold choline-aCSF (still under constant carbogenation), in order to cool it down.

\subsubsection{Transfer the brain onto a filter paper previously wet with aCSF.}

3.2.5. Cut out the brain according to the brain region of interest and preferred angle of slicing. For example, to image the thalamus or the hippocampus on coronal slices, cut out with a scalpel blade the cerebellum and, then, about $2 \mathrm{~mm}$ from the rostral and caudal extremities of the brain.

NOTE: It is important to remove brain parts that are too rostral or too caudal because the smaller the region to trim before reaching the area of interest, the faster the slicing. A total time for slicing (step 3.2.7) of less than $20 \mathrm{~min}$ is recommended.

3.2.6. For coronal slices, position and glue (with cyanoacrylate glue) the caudal face of the brain onto a $10 \mathrm{~cm}$ Petri dish, glued on the cutting block and filled with all the remaining ice-cold choline-aCSF. The block with the dish is, then, positioned in the reservoir chamber of the vibrating slicer, which is positioned in a larger chamber filled with ice.

3.2.7. While keeping constant the $95 \% \mathrm{O}_{2} / 5 \% \mathrm{CO}_{2}$ bubbling of the ice-cold choline-aCSF, cut 300 $\mu \mathrm{m}$-thick coronal slices (speed: $0.08 \mathrm{~mm} \cdot \mathrm{s}^{-1}$, blade vibration: $60 \mathrm{~Hz}$, vibration amplitude: $1 \mathrm{~mm}$ ).

3.2.8. Collect the brain slices with a wide-mouth ( $4 \mathrm{~mm}$ in diameter) disposable transfer pipette, one by one after every single pass of the blade, to avoid the accumulation of toxic components released by the periphery of the slices. Take care to avoid air bubbles during the transfer and place each slice in the choline-aCSF at $32{ }^{\circ} \mathrm{C}$ for about $10 \mathrm{~min}$ for recovery. 
3.2.9. With the transfer pipette, place the slices onto pieces of lens-cleaning paper topped with a drop of choline-aCSF. Aspirate the excess of choline-aCSF and, with the spatula, place the slices, laid on the lens-cleaning tissue, on the mesh of the interface chamber containing carbogenated aCSF at room temperature (see 3.1.3.5). Let the slice recover in this environment for at least 30 $\min$.

NOTE: After this, the slices are ready and can be used for microglia imaging for up to $6 \mathrm{~h}$ after the brain extraction from young (less than one-month-old) mice and up to $4 \mathrm{~h}$ after the brain extraction from two-months-old adults.

\section{Two-photon Microscopy}

\subsection{Parameters setting}

4.1.1. Switch on the multiphoton system (hybrid detectors, laser, scanner, electro-optic modulator, microscope).

4.1.2. Tune the laser at $920 \mathrm{~nm}$, check that the laser is mode-locked, and set the power at $5 \%$ $15 \%$ and the gain at $10 \%$. This corresponds to a power of $3-5 \mathrm{~mW}$ under the objective. Ensure that the nondescanned detectors are engaged and the appropriate emission and excitation filters installed.

4.1.3. Set parameters of the imaging software to the following values: for the frame size, $1024 \mathrm{x}$ 1024 pixels corresponding to an area of $295.07 \times 295.07 \mu \mathrm{m}$; for the zoom, 2 . If the signal is very noisy, apply a line average of 2 . For the pixel dynamics, set the imaging software at 12 bits or more.

NOTE: Images with a higher bit value allow researchers to distinguish smaller differences in fluorescence intensity than images with a lower bit value: a change of one gray value in an 8-bit image would correspond to a change of 16 gray values in a 12-bit and of 256 gray values in a 16bit image. Therefore, higher-bit images are more appropriate for quantitative analysis, but as their size increases with bit depth, storage capacity, and computing power can become limiting.

\subsubsection{Select the scan mode XYZT with a Z-interval range at $2 \mu \mathrm{m}$ and a T-interval of $2 \mathrm{~min}$.}

NOTE: The $x, y$ and $z$ resolution are determined by the Nyquist sampling theorem. A Z-step size around 0.8 would be optimal to resolve microglia processes (with a diameter of $<1 \mu \mathrm{m}$ ), but the optical resolution of multiphoton microscopy is limiting (at $920 \mathrm{~nm}$ with a $0.95 \mathrm{NA}$ objective, the axial resolution is around $1 \mu \mathrm{m}$ ). On top of that physical barrier, in a live-imaging experiment, the sensitivity or signal-to-noise ratio, the resolution, the speed, and the total observation time matter. Taking into account all these parameters, a z-step of $2 \mu \mathrm{m}$ (as in numerous studies ${ }^{3,11,14}$ ), an image size of $1024 \times 1024$ pixels, and a high-speed acquisition using a resonant scanner coupled to HyD detectors (it takes around $15 \mathrm{~s}$ to acquire $50 \mathrm{z}$-plans) were selected here. The frequency of acquisitions is one XYZT series every $2 \mathrm{~min}$ and the total duration is $30 \mathrm{~min}$. If the 
set-up is not fast or sensitive enough, it is possible to reduce the lateral resolution (down to 512 $x$ 512) or the number of z-slices (by imaging exclusively in the $z$-depth which exhibits the strongest fluorescence [i.e., not the deepest z-slices where fluorescence is faint]), or to decrease the speed of the scanner. The axial resolution can also be decreased by increasing the z-step up to $3 \mu \mathrm{m}$, but as this may impact the quantification, all experiments to be compared should be performed with the same z-step.

NOTE: It is possible to perform similar experiments on slices from CX3CR1creER-YFP mice ${ }^{18}$, a mouse line used to induce genetic deletion in microglia only, and in which microglia constitutively express yellow fluorescent protein (YFP). However, the expression level of YFP is very low compared to green fluorescent protein (GFP) in CX3CR1 ${ }^{\mathrm{GFP} /+}$ mice; thus, imaging is possible but challenging and requires the optimization of the acquisition parameters. It is recommended to adjust them as follows.

4.1.5. Tune the laser at $970 \mathrm{~nm}$ (which is better adapted to YFP excitation than $920 \mathrm{~nm}$ ), the power at 50\%, and the gain at 50\%, which corresponds to a laser power under the objective of 5 $-6 \mathrm{~mW}$.

4.1.6. Set a line average of 4 (or more) to improve the signal-to-noise ratio.

\subsection{Positioning of the slice and of the glass micropipette, and the local application of the compound}

4.2.1. Connect the peristaltic pump to the recording chamber, $30 \mathrm{~min}$ before starting the recording. After cleaning the whole perfusion system with $50 \mathrm{~mL}$ of ultrapure water, start the perfusion of the recording chamber with aCSF $(50 \mathrm{~mL})$ contained in a glass beaker under constant carbogenation. Throughout the experiment, keep the circulating aCSF to $32{ }^{\circ} \mathrm{C}$ with an inline microheater or a Peltier heater.

NOTE: A specific perfusion chamber with top and bottom perfusion is designed to optimize the oxygenation on both sides of the slice. The perfusion chamber is composed of two perfectly fitting parts, with a polyamide mesh stretched between them (Figure 2A,B). Compared with other types of chambers, where the slice is directly laying on a glass coverslip, this chamber reduces neuronal death in the bottom part of the slice, improves viability, and reduces the slice movements induced by its swelling.

4.2.2. With a wide-mouth disposable transfer pipette, transfer the brain slice to be imaged to the aCSF beaker to remove the lens paper, let it sink (as a proof that no air bubble is attached), and transfer it to the recording (perfusion) chamber.

4.2.3. Position a slice holder (a hairpin made of platinum with the two branches joined by parallel nylon threads) on the slice to minimize slice movement due to the perfusion flow. 
4.2.4. Use the bright-field illumination to target the brain region of interest (exposure time: 50 to $80 \mathrm{~ms}$ ) using a low magnification objective (5X or 10X). Switch to the higher magnification ( $25 \mathrm{X}$ with a $0.35 \mathrm{X}$ lens) water immersion objective and adjust the position.

NOTE: Avoid to image fields close to the slice holder's nylon threads as they can block the light and locally deform the slice. Make sure that the area of interest is flat. If necessary, remove the slice holder in order to reposition the slice and/or the slice holder.

4.2.5. Use the fluorescence illumination to locate fluorescent microglial cells to be imaged in the field (exposure time: 250 - $500 \mathrm{~ms}$ ).

NOTE: This step allows researchers to check the presence of cells in the region of interest and their fluorescence intensity, and to control for the amount of cellular debris.

4.2.6. Backfill the pipette with $10 \mu \mathrm{L}$ of aCSF with ATP, $5-\mathrm{HT}$, or the drug of interest at its final concentration. Point the tip downward and gently shake the drug-filled pipette to remove any air bubbles trapped in the tip.

NOTE: If the solution to be injected tends to form bubbles, consider using borosilicate pipettes with an internal filament. Leakage of ATP out of the pipette can attract microglial processes even before the injection (if this occurs, it will be visible at the analysis step). Although this should be moderate with the ATP concentration used $\left(500 \mu \mathrm{mol} \cdot \mathrm{L}^{-1}\right)$, if it is an issue, consider prefilling the micropipette with $2 \mathrm{~mL}$ of aCSF prior to adding the ATP (or other compound) solution at step 4.2.6.

4.2.7. Mount the filled pipette in a pipette holder, connected with transparent tubing to a $5 \mathrm{~mL}$ syringe, with a plunger positioned at the $5 \mathrm{~mL}$ position. The pipette holder itself is mounted onto a three-axis micromanipulator.

4.2.8. Under bright-field illumination, use the micromanipulator to position the pipette in the center of the field. For a reproducible and optimal centering, display and use the rulers on the image.

4.2.9. Lower the pipette gently toward the slice, controlling and adjusting the objective at the same time, until the pipette tip lightly touches the surface of the slice. Stopping the descent of the pipette as soon as it is visible that the slice has been touched allows the pipette tip to penetrate $80-100 \mu \mathrm{m}$ of the surface of the slice (see Figure 3B).

4.2.10. Tune the laser (see the parameters above) and switch the microscope to the multiphoton mode. Make sure that the chamber is screened from any light source (e.g., a computer screen). Switch on the nondescanned detectors and set the gain. Use a lookup table (LUT) with a colorcoded upper limit to avoid saturating the pixels in the image. 
4.2.11. Determine the thickness of the slice to be imaged (i.e., the upper and lower z-positions where fluorescence is detectable [usually between 220 and $290 \mu \mathrm{m}$ in total]).

NOTE: At the surface of the slice, there is an increased density of processes and possibly of microglia, often with an unusual morphology, in comparison with the inside of the slice. This accumulation will be more striking with time (i.e., more visible in the last than in the first brain slice to be imaged). Therefore, the z-planes in the first $\sim 30 \mu \mathrm{m}$ should not be used for the analysis and can even be skipped for the acquisition.

4.2.12. Start recording for a total duration of $30 \mathrm{~min}$ (or more if desired) and after a $5 \mathrm{~min}$ baseline, locally apply the compound to be tested (without interrupting the imaging). To do this, slowly press the plunger of the syringe connected to the micropipette, from the $5 \mathrm{~mL}$ to the $1 \mathrm{~mL}$ position (in about $5 \mathrm{~s}$ ). Resistance when pressing the plunger must be felt immediately. If not, the tip might be broken.

NOTE: For a trained experimenter, the injections with this method are reproducible, but alternatively to the manual manipulation of a syringe, the pipette could be linked to an automated pressure ejection system to allow a better control of the volume delivered. The injection creates a physical distortion of the slice at the site of the injection. This distortion is visible $a$ posteriori in the first two or three images after the injection but should not be visible on the fourth image, (i.e., 8 min after the injection). If it persists, consider changing the parameters for the pipette preparation.

4.2.13. At the end of the acquisition ( $30 \mathrm{~min}$ ), discard the micropipette and remove the slice. If desired, fix the slice for further immunolabeling. For example, the SNAPSHOT method is optimized for the fixation and staining of thick slices ${ }^{23}$.

4.2.14. Prior to starting to image a new slice, make the 2D movie (section 5.1) in order to check that microglia have a normal morphology and are moving and, thus, that the slices are healthy.

\section{Analysis of the Attraction of Microglial Processes}

\subsection{D projection and drift correction}

\subsubsection{Open the file (.LIF) with $\mathrm{Fiji}^{24}$.}

5.1.2. If necessary, make a substack (Image/Stacks/Tools/Make Substack) with only the z-planes of interest. For example, exclude the z-planes corresponding to the surface of the slice if they have been acquired but are not to be used for the analysis (see the NOTE after step 4.2.11) and the deepest z-planes with no fluorescence. The final stack generally contains 90 - 110 z-slices (180 - $220 \mu \mathrm{m}$ ).

5.1.3. Launch the $\mathbf{Z}$ project function (Image/Stacks/Z Project") and select the Max Intensity projection type to make the projections of the z-stack acquired at each time point. 
439

440

441

442

443

444

445

446

447

448

449

450

451

452

453

454

455

456

457

458

459

461

460

462

463

464

465

466

467

468

469

470

471

472

473

474

475

476

477

478

479

5.1.4. Launch the MultiStackReg plugin (Plugin/Registration/MultiStackReg), selecting Action 1: Align and Transformation: Rigid Body to correct slight drifts that may have occurred during the acquisition. Save this 2D movie as a new file (.TIFF).

\subsection{Data processing}

5.2.1. Open this new file with Icy ${ }^{25}$.

5.2.2. Draw a circular $\mathbf{R} \mathbf{1}$ region of interest (ROI) of $35 \mu \mathrm{m}$ in diameter, centered on the injection site (identified notably by the shadow of the pipette and the distortion created at the time of injection).

5.2.3. Use the plugin ROI intensity evolution and measure the mean intensity over time in R1.

5.2.4. Save the results to an .XLS file.

\subsection{Quantification and representation of the results}

5.3.1. To quantify the microglial response over time, determine at each time point

$$
R(t)=\frac{[R 1(t)-\bar{R} 1(0)]}{\bar{R} 1(0)}
$$

Here, $\bar{R} 1(0)$ is the mean of the $R 1(t)$ values before the injection. Then, the results can be represented as a kinetic of the microglial response, or at a specific time point (see Figure 7).

\section{REPRESENTATIVE RESULTS:}

This protocol describes a method to induce, observe, and quantify the oriented growth of microglial processes toward a locally applied compound, for example, ATP or 5-HT, in acute brain slices from young or adult (at least up to two-month-old) mice. Among the factors that contribute to maintaining brain slices from adult animals in a healthy state for several hours is the use of two tools designed to optimize cell survival at two steps of the protocol. First, the interface slice holder in the interface chamber (Figure 1) improves the conservation of the slices after cutting. Second, the recording chamber (Figure 2) has a perfusion system which allows the aCSF to run both at the top and at the bottom of the slice during imaging. The recording chamber dimensions used here are set to fit standard microscopes as they are similar to classical bath chamber inserts (with a 62 mm outer diameter), but as their models are downloadable from the Supplemental Material, the design can be adapted to fit in slice holders of other dimensions. To note is that each chamber is made by the assembly, without glue, of two perfectly fitting parts, with a polyamide mesh stretched between them. 
To deliver ATP or 5-HT in a small area and induce a local response of microglia, a pipette containing the compound (visible in Figure $\mathbf{2 C , D}$ ) is placed with its tip on top of the slice. In the first experiments, it can be helpful to add a fluorescent dye to the solution, in order to visualize the position of the pipette tip on the images that are acquired with the two-photon microscope (Figure 3A). In Figure 3B, it is visible that although the experimenter stopped the pipette descent as soon as its tip touched and deformed the slice surface on the bright-field image of the computer screen, its thin extremity entered slightly into the tissue, down to $80-100 \mu \mathrm{m}$ from the surface. It is important that the pipette is not too superficial because it may not deliver the solution correctly onto the cells, nor enter too deep because it may reach a region where the fluorescence signal is too low. The parameters that may affect the depth reached by the pipette tip are the angle of the pipette, which can be adjusted with the three-axis micromanipulator, and the pipette mouth diameter.

Note that, with the projection along the Y-axis (Figure 3B), it is possible to observe that the fluorescence is stronger in the upper than in the lower part of the slice. This is due both to the progressive blunting of the signal inside the slice and to the fact that processes of microglia of the superficial layers of the slice, and some cell bodies, tend to migrate toward the surface. As a result, microglia in the most superficial layers of the slice might show a different morphology than those inside the slice, indicating that they are not in the same state, and may react differently to stimulation. Thus, superficial microglial activation may mask, in the z-projection, the response of the microglia below. In addition, the surface is often tilted, and the first z-images of the stacks are patchy. Therefore, we recommend, for a better accuracy, to exclude the most superficial z-planes from the z-projection and analysis. However, as altered microglia morphology and the presence of debris in the superficial layers are indicators of the condition of the slice, it can be interesting to image the full depth of a slice to check its status a posteriori. This can be especially useful to new experimenters who may not easily recognize abnormal microglia or debris from single zplanes. The z-planes taken in the first $30 \mu \mathrm{m}$ will then be excluded at the z-projection step (step 5.1.2 of the protocol).

Once the slice has been treated with the compound of interest and recorded, the series of zplanes (excluding the first $30 \mu \mathrm{m}$, as discussed above) imaged at each time point are projected along the $z$-axis to make a $2 \mathrm{D}$ movie of $z$-projection images. To note is that in the protocol presented here, the thickness used for the max z-projection encompasses all the z-slices where fluorescence is visible (usually $180-220 \mu \mathrm{m}$, see step 5.1 .2 of the protocol). Therefore, variations in the absolute number of $z$-slices do not impact the quantification of the response. In contrast, some studies use thinner z-stacks $(40-60 \mu \mathrm{m})$ for z-projection ${ }^{6,7,11,27}$. This is another option, which comes with the risk to exclude some z-slices which exhibit a response, as we observed that the attractant effect was visible as far as $70 \mu \mathrm{m}$ (in z) away from the pipette tip in some experiments. If the thinner option is preferred, it is, thus, critical to center the z-stack on the pipette tip in $z$, and importantly, only z-projections done in the same manner (i.e., using all fluorescent z-slices or using a thin z-stack) can be compared.

An R1 region of interest is then defined for quantitative analysis. Figure 4 shows an ROI on a zprojection. The red dashed lines represent the putative position of the pipette. The yellow circle 
is R1, drawn with Icy, and centered on the putative tip of the pipette. Importantly, we observed that a noticeable variability arose during the quantification from the localization of the R1 ROI, whose misplacement leads to an underestimation of the response. To help positioning the ROI on the site of delivery, we recommend displaying the rulers in the acquisition software when positioning the pipette in the brightfield, in order to place the pipette tip always in the same central $X Y$ position in the field to be imaged. This provides an indication of where to position, later, the ROI in the Z-projection of the fluorescence images. However, the final tip position and, thus, the actual site of delivery will be slightly shifted from this central position, depending on the depth reached by the pipette tip. For this reason, to position R1, we take into consideration three other criteria: (i) R1 must be at the tip of the pipette, the position of which is inferred from the dark background in the lower right corner, (ii) it should correspond to the area where a transient distortion of the tissue occurs when the compound is injected, and (ii) it should correspond to the area where a local response (if any) is observed. If this is not sufficient to locate with good accuracy the point of delivery, filling the pipette with a fluorescent compound will help. After having drawn R1, we run the movie again to check that it is well positioned for all the time points and that there are no aberrant drift, distortion, or artifact fluorescence at any time point, which could bias the quantification. The movie corresponding to Figure 4, and illustrating the effect of ATP application, can be found in the supplemental material (Movie S1).

We initially studied the attraction of microglial processes toward ATP or 5-HT in the thalamus of P11 mice ${ }^{15}$. More recently, these experiments were repeated in slices from four-day- to twomonth-old mice, and in various regions (for example, Figure $\mathbf{3}$ corresponds to a recording in the hippocampus). We mostly used three- to four-week-old mice, which combines the advantages of having a mature brain-with mature and ramified microglia-with a good slice viability. Importantly, representative normal-state microglia are characterized by a small soma, long processes with small terminals, and constantly moving processes at the baseline condition. Typically, in slices from 18- to 30-day-old mice, the microglia morphology remains mostly unchanged for up to $6 \mathrm{~h}$ after the slice preparation. For slices from older mice (two months old), the ability to maintain the slices in a physiological state is reduced $(\sim 4 \mathrm{~h})$. Examples of microglial direction motility in the thalamus, in response to $5-\mathrm{HT}$ in a slice from a 20-day-old mouse and to ATP in a slice from a two-month-old mouse, are provided in Figure 5. The movie corresponding to the 5-HT application in Figure $\mathbf{5}$ can be found in the supplemental material (Movie S2).

Figure 6 presents two suboptimal experiments made on slices kept for longer than $6 \mathrm{~h}$ in aCSF before they were imaged (only times 0 are shown). In Figure 6A, note the presence of microglia with multiple short processes or with enlarged terminals reminiscent of neurite growth cones, and the presence of many fluorescent cell debris or particles. These very fluorescent elements stay immobile or move randomly during imaging, indicating that they do not belong to "living" microglia (see Movie S3 in the supplemental material). To note is that, in this particular example, microglia were constantly scanning their environment, but their morphology was so altered that they could not be considered as being in a physiological state. Moreover, the large amount of debris would have made the fluorescence analysis poorly reliable. Figure 6B illustrates the presence of microglia with large and flat cell bodies or protrusions, which can sometimes be found on the superficial layers of slices that have been kept several hours before they were 
imaged. Only superficial z-positions have been used to make this z-projection. Figure $6 \mathrm{C}$ is a substack, at deeper z-positions, from the same brain slice as shown in Figure 6B. Although they are not positioned at the surface, these microglia have an abnormal "bushy" aspect. Due to the aspect of microglia, the movies corresponding to these two slices were not used for quantification.

After extracting the data from the 2D-movies, the results can be represented by plotting normalized $R(t)$ fluorescence over time. A graph summarizing several experiments is presented in Figure $\mathbf{7 A}$, to show the variability of the responses. Note that the fluorescence decreases immediately but transiently (in the first three images) after the injection due to tissue distortion (i.e., the liquid injection with the pipette transiently pushes the tissue away, and then increases). The variability in the response to ATP illustrates the fact that even when slices look similarly healthy, with motile and ramified microglia, they do not respond in the same manner (but all of them do respond). On top of intrinsic sample heterogeneity, there is some looseness in the R1 positioning, as mentioned above, and we also cannot exclude an impact of, for example, differences in the volume of the solution which is delivered. Therefore, to detect small effects or variations, it may be interesting to use an automatic device for the compound injection.

Figure 7B shows how the size of the ROI also impacts the quantification, here of the growth of ATP-induced processes. Increasing the diameter from 35 (the diameter used in Figure 7A and for all the analyses presented here) to 50 or $70 \mu \mathrm{m}$ reduces the variability among experiments (slices) by suppressing the issue of the small R1 positioning. However, it also decreases accuracy and the magnitude of the detected response. Indeed, with larger ROIs, there is more background due to processes or cell bodies not affected by the treatment, and a growth of processes can be partially blunted by the concomitant retraction of microglial branches more distant from the pipette but nevertheless inside the ROI. In conclusion, it can be relevant to use ROIs with a different diameter circle than one that is $35 \mu \mathrm{m}$, but it is fundamental that the ROI is always the same in all the data sets to be compared.

Figure 7C shows the mean \pm SEM for several experiments with aCSF, ATP, or 5-HT. The effect of ATP is in the same range as those obtained by other groups with a similar method in vivo $(0.5$ according to Davalos et $a l_{.}^{3}$ and 0.4 according to Haynes et $a l^{13}$ ) and in slices (0.8 if normalized as is done here, according to Dissing-Olesen et al. ${ }^{5}$, and 0.6 according to Pagani et al. ${ }^{28}$ ). On the one hand, differences can come from biological parameters, such as the slice preparation method, the amount of ATP injected, the age of the mouse, or the brain region used, and on the other hand from analysis parameters, such as the diameter of the ROI and the thickness of the zstacks used for the z-projection (i.e., the whole thickness where fluorescence is detected, or only the $40-60 \mu \mathrm{m}$ around the pipette tip, where the maximal response is expected).

In Figure 7C, the aCSF injection is the negative control, necessary to check that the local injection and the pressure of the pipette do not cause an injury that may attract microglial processes. In addition, the aCSF control allows researchers to check that there is no photobleaching over time. Indeed, photobleaching, the photon-induced destruction of the fluorescent proteins or fluorophores, would have induced a progressive decrease of fluorescence over time. As it can bias 
measurements, it is important to rigorously check that there is no photobleaching in the experimental conditions. To do this, it is recommended to acquire an XYZT series on a slice with GFP-expressing microglia, for $30 \mathrm{~min}$ (aCSF can be injected but, actually, no stimulation is needed), with the excitation and acquisition parameters set as in the experimental conditions. Then, a quantitative measure of the fluorescence over time in different regions of interest, including microglia cell bodies or processes, will reveal if there is a gradual loss in emission intensity, usually an exponential decay, indicating photobleaching. If this is the case, some adjustments can be performed: a realignment of the laser, a reduction of the laser power and an increase of the detector gain, a reduction of the number of z-planes, and an increase of the interval between them to limit illumination. Photobleaching is favored by high-power or long (ex: repeated illumination for line averaging) excitation; thus, researchers must pay attention to it if a sustained illumination is used to image cells with low fluorescence.

After the local injection of ATP or 5-HT, there is an increase of fluorescence in R1, which reaches a plateau (Figure $\mathbf{7 C}$ ). In addition to kinetics, it can be interesting to compare the attraction at a stable endpoint. Here, we chose to represent the microglial response at $t=26 \min$ (i.e., $20 \mathrm{~min}$ after the injection) in Figure 7D. This is useful to statistically compare compounds, or to test the effect of antagonists which can be added in the bath (i.e., in the perfusion solution, or together with the compound in the pipette [not shown]).

\section{FIGURE LEGENDS:}

Figure 1: Interface chamber details. (A) Outline for the 3D printing of the slice holder. The external diameter of the holder is $7 \mathrm{~cm}$. (B) Interface slice holder with the nylon mesh (arrow) that allows researchers to keep slices at the liquid-air interface. (C) Interface chamber device that makes it possible to maintain the slices in a carbogenated (the arrow indicates the tubing for bubbling) and humidified environment before they are imaged.

Figure 2: Perfusion chamber details. (A) Outline for the 3D printing. The external diameter of the perfusion chamber is $5.9 \mathrm{~cm}$. (B) The perfusion chamber with the nylon mesh (arrow) that supports the slice, prevents it from touching the slide below, and allows the aCSF to flow above and under it. (C) Picture of the assembly at the two-photon microscope. The micropipette which will deliver the compound locally is visible on the right (asterisk). (D) Pipette imaged in brightfield. The scale bar $=60 \mu \mathrm{m}$.

Figure 3: Position of the micropipette in the slice. Example of an acquisition of a stack of images (in the hippocampus of a 30 -day-old animal) with a micropipette filled with fluorescein $(1 \mu \mathrm{M})$. Fluorescein makes it possible to locate the pipette (asterisk) in the max projections along (A) the $\mathbf{z}$-axis or (B) the $\mathbf{y}$-axis. Note in panel $\mathbf{B}$ that, although fainter in fluorescence, there are also microglia below the pipette tip. In this illustrated example, the full stack, including the images taken in the most superficial part of the slice, has been used for the projections. The scale bar = $30 \mu \mathrm{m}$ in panel $\mathbf{A}$ and as indicated (each graduation $=50 \mu \mathrm{m}$ ) in panel $\mathbf{B}$. The $z$-thickness of the stack $=220 \mu \mathrm{m}$. 
Figure 4: Positioning of R1, the ROI used for quantification. This figure shows three experimental time points of an experiment where ATP has been applied on a slice (from the thalamus of a 20day-old animal), with drawings of the putative location of the pipette (red dashed line on the first time point) and the delineation of the R1 ROI. The scale bar $=30 \mu \mathrm{m}$. Note the darker area in the lower right corner, corresponding to the shade of the pipette, which interferes with illumination and imaging. Note, also, the small region of intense fluorescence at $25 \mathrm{~min}$, which indicates the location of the pipette tip. The z-thickness of the stack $=220 \mu \mathrm{m}$.

Figure 5: Microglial responses. Responses to the local application of 5-HT (5 $\mu \mathrm{M})$ on a brain slice from a 20-day-old mouse (left; the slice z-thickness $=220 \mu \mathrm{m})$ and of ATP $(500 \mu \mathrm{M})$ on a slice from a two-month-old mouse (right; the slice z-thickness $=220 \mu \mathrm{m}$ ). The red dashed lines represent the pipette position. Both recordings have been done in the thalamus, and the upper $30 \mu \mathrm{m}$ of the slices have been excluded. The images are taken before (upper row) and $25 \mathrm{~min}$ after (lower row) the injection. The scale bar $=30 \mu \mathrm{m}$.

Figure 6: Examples of suboptimal experiments. These slices have waited for more than $6 \mathrm{~h}$ before imaging and have been imaged since their upper surface. (A) Example of a z-stack with a lot of debris (roughly round, fluorescent particles, but with irregular borders; asterisks) and "bushy" microglia (arrows), that is, with numerous but short processes. Note the enlarged process terminals (arrowheads) which look like axonal growth cones. The z-thickness of the stack $=220$ $\mu \mathrm{m}$. The other two panels show two sub-stacks of the same slice, with (B) 1-30 z-planes (zthickness $=59 \mu \mathrm{m})$ and $(C)$ 30-120 z-planes $(z$-thickness $=180 \mu \mathrm{m}$ ). On the top planes (shown in panel $\mathbf{B}$ ), in addition to the large process terminals and debris like in panel $\mathbf{A}$, there are microglia with unusual large flat bodies or protrusions (stars). On the deeper planes (shown in panel $\mathbf{C}$ ), there is no debris nor flat objects, but the cells are bushy (arrows) and the density is unusually high. Altogether, these observations indicate that the microglia are not in a normal state.

Figure 7: Quantification of the attraction of microglial processes. (A) Example of a series of experiments with ATP to illustrate variability. (B) Impact of the ROI size on the quantification. Each experiment shown in panel A (ATP injection) has been analyzed with an ROI of 35, 50, or $70 \mu \mathrm{m}$ diameter. The mean \pm SEM of the fluorescence at each time point is shown for the different ROIs. (C) Summary of experiments with aCSF, 5-HT, and ATP. The aCSF injection has no effect on the location of microglial processes. ATP and 5-HT induce a localized growth of microglial processes toward the pipette, measured with the local increase of fluorescence. The mean \pm SEM are indicated. (D) Summary of microglial responses at $t=20 \mathrm{~min}$ postinjection $(26 \mathrm{~min}$ from the beginning of recording). The mean \pm SEM are indicated. One-way ANOVA with Dunnett post hoc test is used. ${ }^{* *} p<0.01, * * * p<0.001$ compared to the aCSF injection. For aCSF, $n=7$; for ATP, $n$ $=8$; for $5-\mathrm{HT}, n=6$. All these experiments were performed in the thalamus, but similar results can be obtained from the hippocampus or cortex. The z-thickness of the stacks used for z-projections and quantification $=180-220 \mu \mathrm{m}$. All measures except those in panel $\mathbf{B}$ have been done with a $35 \mu \mathrm{m}$-diameter ROI.

Movie S1: Example of the effect of a local ATP $(500 \mu \mathrm{M})$ application. This experiment has been performed in the thalamus of a 20-day-old animal. Images from this movie have been used for 
Figure 4. The scale bar $=30 \mu \mathrm{m}$.

701

702

703

704

705

706

707

708

709

710

711

712

713

714

715

716

717

718

719

720

721

722

723

724

725

726

727

728

729

730

731

732

733

734

735

736

737

738

739

740

741

742

743
Movie S2: Example of the effect of a local 5-HT (5 $\mu \mathrm{M})$ application. This experiment has been performed in the thalamus of a 20-day-old animal. Images from this movie have been used for Figure 5 (left). The scale bar $=30 \mu \mathrm{m}$.

Movie S3: Example of a suboptimal experiment in a slice having waited for more than $\mathbf{6} \mathrm{h}$ before imaging. Note the presence of numerous debris which moves randomly over time and the unusual morphology of the microglia. An image from this movie is used as Figure 6A. The scale bar $=30 \mu \mathrm{m}$.

\section{Supplementary File. Imaging chamber and Interface holding chamber}

\section{DISCUSSION:}

By maintaining, unlike in dissociated or organotypic slice culture, a structural integrity with limited network adjustments, acute brain slices allow researchers to study microglia in their physiological environment. However, one of the major limitations is the fact that the slicing procedure creates injuries that can rapidly compromise the viability of neurons, particularly in the adult brain. As microglia are particularly reactive to cell damage, it is important to limit neuronal cell death as much as possible to preserve microglia close to their physiological state. This, in turn, contributes via a virtuous circle to a better general condition of the slice.

The protocol described in this article applies several improvements found in the literature for electrophysiology experiments, aimed at obtaining a better viability of the slices over several hours, especially from adult mice. To overcome the age-related disparity in slice viability, we substituted sodium with choline and used this choline-aCSF medium during the cardiac perfusion, slicing, and recovery phases. This brings the cell excitotoxicity to a minimum ${ }^{29,30}$. It should be noted that, in addition to choline-aCSF, there are many other alternative aCSF recipes that are highly effective for neuronal preservation, like N-methyl-D-glucamine (NMDG)-aCSF ${ }^{31}$, which could also be interesting for microglial studies but have not been tested here. Another critical step in this procedure is the cardiac perfusion. By perfusing the animal with a cold choline-aCSF solution, containing low $\mathrm{Na}^{+}$, low $\mathrm{Ca}^{2+}$, and high $\mathrm{Mg}^{2+}$, a rapid decrease in body temperature is induced and the metabolic activity of the cells in the brain is lowered, thereby reducing the cellular stress caused by the cutting. Indeed, we observed that this approach offered more protection than simply removing the whole brain and submerging it in aCSF solution. Inspired by a protocol optimized for patch-clamp and optogenetic studies" ${ }^{29}$, we also used a "protective recovery" period immediately following the physical slicing, allowing the slices to recover from the trauma of slicing for $10 \mathrm{~min}$ in choline-aCSF at $32{ }^{\circ} \mathrm{C}$. Afterward, slices were transferred into the interface holding chamber to recover for an additional minimum time of $30 \mathrm{~min}$. The duration of this second recovery period could be optimized according to the brain area of interest, the $\mathrm{Na}^{+}$ substitute, and the age of the animal, and it should last at least $1 \mathrm{~h}$ if electrophysiology has to be performed in parallel with imaging.

The maintenance of the slice in a healthy state before use and the perfusion during imaging are 
also important parameters. Both the interface and the dual-perfusion chamber are widely established tools in electrophysiology. For example, the interface method has been used to improve the viability of hippocampal slices since $1995^{26}$. Devices similar to the chambers used here are proposed by several companies but not yet commonly used for microglia imaging. We designed an interface holding chamber, where the slices lay on a net and are on the interface with a large reservoir of aCSF, delaying the cellular deterioration of the slices during prolonged incubation times. In the imaging chamber, we used a designed double-sided perfusion chamber which allows increased oxygenation of acute slice preparations, maintaining the cells in a healthy state during multiphoton microscopy. In contrast with the single perfusion, the double perfusion of submerged slices increases the viability of the cells, since oxygen, as well as other materials, can freely and effectively diffuse with a high flow rate from both sides of considerably thick slices.

This protocol is aimed at quantifying the global attractant effect of ATP or other compounds on microglial processes, and its quantification method is based on the one used in Davalos et al. ${ }^{3}$. It is possible to perform other kinds of analysis. For example, an alternative method, which does not depend on the fluorescence level of the microglial processes but requires more actions of the experimenter for the image analysis, is to measure the reduction of the empty space around the pipette tip after compound application ${ }^{11,32}$. It is also possible to track the movement of the tip of individual microglial processes ${ }^{15,28}$.

In addition, the isotropic motility or surveillance of microglia can also be registered in basal condition or upon the bath application of compounds of interest on the slices prepared with this protocol. However, to quantify the fast extension and retraction rates of microglial processes and detect potential variations, it would be relevant to increase the acquisition frequency. For example, Pagani et al. use a frequency of one image every $10 \mathrm{~s}^{28}$.

Finally, recent publications described methods to quantify morphological changes or the motility of individual processes in three dimensions. For such analyses, although a z-step interval of $2 \mu \mathrm{m}$ is enough for some programs $\mathrm{s}^{27}$, others require a better axial resolution (i.e., a z-step of $0.4 \mu \mathrm{m}$ according to Heindl et al. ${ }^{33}$.

Here, we showed experiments on wild-type mice with an endogenous expression of GFP in microglia and with a mechanical application of compounds, but this protocol can be adapted to other fluorescent reporter proteins in microglia, like YFP ${ }^{18}$, or to the exogenous labeling of microglia with fluorescent isolectin ${ }^{11,34}$. Finally, we have studied the outgrowth of microglial processes toward a local application of ATP or 5-HT in a wild-type normal environment, but this protocol could be used to test other compounds, other kinds of stimulation (e.g., caged compounds), and to test how directional motility is affected by mutations, pharmacological agents, or pathological contexts.

\section{ACKNOWLEDGMENTS :}

This work has been supported in part by the Centre National de la Recherche Scientifique, the Institut National de la Santé et de la Recherche Médicale, the Sorbonne Université Sciences, and by grants from Sorbonne Universités-Pierre et Marie Curie University (Emergence-UPMC program 
2011/2014), the Fondation pour la Recherche sur le Cerveau, the Fondation de France, the Fondation pour la Recherche Médicale "Equipe FRM DEQ2014039529", the French Ministry of Research (Agence Nationale pour la Recherche ANR-17-CE16-0008 and the Investissements d'Avenir programme "Bio-Psy Labex" ANR-11-IDEX-0004-02) and a Collaborative Research in Computational Neuroscience program, National Science Foundation/French National Agency for Research (number : 1515686). All the authors are affiliated to research groups which are members of the Paris School of Neuroscience (ENP) and of the Bio-Psy Labex. F.E. is a Ph.D. student affiliated with Sorbonne Université, Collège Doctoral, F-75005 Paris, France, and is funded by the Bio-Psy Labex. V.M. is a post-doctoral fellow funded by the Collaborative Research in Computational Neuroscience program, National Science Foundation/French National Agency for Research (number: 1515686). The authors thank Marta Kolodziejczak who participated in the initiation of the project.

\section{DISCLOSURES:}

The authors have nothing to disclose.

\section{REFERENCES:}

1. Salter, M. W., Stevens, B. Microglia emerge as central players in brain disease. Nature Publishing Group. 23 (9), 1018-1027, doi:10.1038/nm.4397 (2017).

2. Tay, T. L., Savage, J., Hui, C. W., Bisht, K., Tremblay, M.-È. Microglia across the lifespan: from origin to function in brain development, plasticity and cognition. The Journal of Physiology doi:10.1113/JP272134 (2016).

3. Davalos, D. et al. ATP mediates rapid microglial response to local brain injury in vivo. Nature Neuroscience. 8 (6), 752-758, doi:10.1038/nn1472 (2005).

4. Nimmerjahn, A. Resting Microglial Cells Are Highly Dynamic Surveillants of Brain Parenchyma in Vivo. Science. 308 (5726), 1314-1318, doi:10.1126/science.1110647 (2005).

5. Dissing-Olesen, L. et al. Activation of neuronal NMDA receptors triggers transient ATPmediated microglial process outgrowth. The Journal of Neuroscience: The Official Journal of the Society for Neuroscience. 34 (32), 10511-10527, doi:10.1523/JNEUROSCI.0405-14.2014 (2014).

6. Gyoneva, S., Traynelis, S. F. Norepinephrine modulates the motility of resting and activated microglia via different adrenergic receptors. Journal of Biological Chemistry. 288 (21), 1529115302, doi:10.1074/jbc.M113.458901 (2013).

7. Eyo, U. B. et al. Neuronal hyperactivity recruits microglial processes via neuronal NMDA receptors and microglial P2Y12 receptors after status epilepticus. The Journal of Neuroscience: The Official Journal of the Society for Neuroscience. 34 (32), 10528-10540, doi:10.1523/JNEUROSCI.0416-14.2014 (2014). 
8. Hristovska, I., Pascual, O. Deciphering Resting Microglial Morphology and Process Motility from a Synaptic Prospect. Frontiers in Integrative Neuroscience. 9, 1231, doi:10.1152/jn.01210.2007 (2016).

9. Avignone, E., Lepleux, M., Angibaud, J., Nägerl, U. V. Altered morphological dynamics of activated microglia after induction of status epilepticus. Journal of Neuroinflammation. 12, 202, doi:10.1186/s12974-015-0421-6 (2015).

10. Abiega, O. et al. Neuronal Hyperactivity Disturbs ATP Microgradients, Impairs Microglial Motility, and Reduces Phagocytic Receptor Expression Triggering Apoptosis/Microglial Phagocytosis Uncoupling. PLoS Biology. 14 (5), e1002466, doi:10.1371/journal.pbio.1002466 (2016).

11. Madry, C. et al. Microglial Ramification, Surveillance, and Interleukin-1 $\beta$ Release Are Regulated by the Two-Pore Domain K+Channel THIK-1. Neuron. 97 (2), 299-312.e6, doi:10.1016/j.neuron.2017.12.002 (2018).

12. Honda, S. et al. Extracellular ATP or ADP induce chemotaxis of cultured microglia through Gi/o-coupled P2Y receptors. The Journal of Neuroscience: The Official Journal of the Society for Neuroscience. 21 (6), 1975-1982 (2001).

13. Haynes, S. E. et al. The P2Y12 receptor regulates microglial activation by extracellular nucleotides. Nature Neuroscience. 9 (12), 1512-1519, doi:10.1038/nn1805 (2006).

14. Wu, L.-J., Vadakkan, K. I., Zhuo, M. ATP-induced chemotaxis of microglial processes requires $\mathrm{P} 2 \mathrm{Y}$ receptor-activated initiation of outward potassium currents. Glia. 55 (8), 810-821, doi:10.1002/glia.20500 (2007).

15. Kolodziejczak, M. et al. Serotonin Modulates Developmental Microglia via 5-HT 2BReceptors: Potential Implication during Synaptic Refinement of Retinogeniculate Projections. ACS Chemical Neuroscience. 6 (7), 1219-1230, doi:10.1021/cn5003489 (2015).

16. Schafer, D. P. et al. Microglia Sculpt Postnatal Neural Circuits in an Activity and ComplementDependent Manner. Neuron. 74 (4), 691-705, doi:10.1016/j.neuron.2012.03.026 (2012).

17. Pfeiffer, T., Avignone, E., Nägerl, U. V. Induction of hippocampal long-term potentiation increases the morphological dynamics of microglial processes and prolongs their contacts with dendritic spines. Scientific Reports. 6, 32422, doi:10.1038/srep32422 (2016).

18. Parkhurst, C. N. et al. Microglia Promote Learning-Dependent Synapse Formation through Brain-Derived Neurotrophic Factor. Cell. 155 (7), 1596-1609, doi:10.1016/j.cell.2013.11.030 (2013). 
19. Wu, Y., Dissing-Olesen, L., Macvicar, B. A., Stevens, B. Microglia: Dynamic Mediators of Synapse Development and Plasticity. Trends in Immunology. 36 (10), 605-613, doi:10.1016/j.it.2015.08.008 (2015).

20. Ohsawa, K. et al. P2Y12 receptor-mediated integrin-beta1 activation regulates microglial process extension induced by ATP. Glia. 58 (7), 790-801, doi:10.1002/glia.20963 (2010).

21. Kurpius, D., Wilson, N., Fuller, L., Hoffman, A., Dailey, M. E. Early activation, motility, and homing of neonatal microglia to injured neurons does not require protein synthesis. Glia. 54 (1), 58-70, doi:10.1002/glia.20355 (2006).

22. Stence, N., Waite, M., Dailey, M. E. Dynamics of microglial activation: a confocal time-lapse analysis in hippocampal slices. Glia. 33 (3), 256-266 (2001).

23. Dissing-Olesen, L., Macvicar, B. A. Fixation and Immunolabeling of Brain Slices: SNAPSHOT Method. Current Protocols in Neuroscience. 71, 1.23.1-12, doi:10.1002/0471142301.ns0123s71 (2015).

24. Schindelin, J. et al. Fiji: an open-source platform for biological-image analysis. Nature Methods. 9 (7), 676-682, doi:10.1038/nmeth.2019 (2012).

25. de Chaumont, F. et al. Icy: an open bioimage informatics platform for extended reproducible research. Nature Methods. 9 (7), 690-696, doi:10.1038/nmeth.2075 (2012).

26. Aitken, P. G. et al. Preparative methods for brain slices: a discussion. Journal of Neuroscience Methods. 59 (1), 139-149 (1995).

27. Paris, I. et al. ProMolJ: A new tool for automatic three-dimensional analysis of microglial process motility. Glia. 66 (4), 828-845, doi:10.1002/glia.23287 (2018).

28. Pagani, F. et al. Defective microglial development in the hippocampus of Cx3cr1 deficient mice. Frontiers in Cellular Neuroscience. 9 (229), 111, doi:10.3389/fncel.2015.00111 (2015).

29. Ting, J. T., Daigle, T. L., Chen, Q., Feng, G. Acute brain slice methods for adult and aging animals: application of targeted patch clamp analysis and optogenetics. Methods in Molecular Biology (Clifton, NJ). 1183, 221-242, doi:10.1007/978-1-4939-1096-0_14 (2014).

30. Mainen, Z. F. et al. Two-photon imaging in living brain slices. Methods (San Diego, CA). 18 (2), 231-239, 181, doi:10.1006/meth.1999.0776 (1999).

31. Tanaka, Y., Tanaka, Y., Furuta, T., Yanagawa, Y., Kaneko, T. The effects of cutting solutions on the viability of GABAergic interneurons in cerebral cortical slices of adult mice. Journal of Neuroscience Methods. 171 (1), 118-125, doi:10.1016/j.jneumeth.2008.02.021 (2008). 
918 32. Gyoneva, S. et al. Systemic inflammation regulates microglial responses to tissue damage in 919 vivo. Glia. 62 (8), 1345-1360, doi:10.1002/glia.22686 (2014).

920

921 33. Heindl, S. et al. Automated Morphological Analysis of Microglia After Stroke. Frontiers in 922 Cellular Neuroscience. 12, 106, doi:10.3389/fncel.2018.00106 (2018).

923

924 34. Dailey, M. E., Eyo, U., Fuller, L., Hass, J., Kurpius, D. Imaging microglia in brain slices and slice 925 cultures. Cold Spring Harbor Protocols. 2013 (12), 1142-1148, doi:10.1101/pdb.prot079483 926 (2013). 


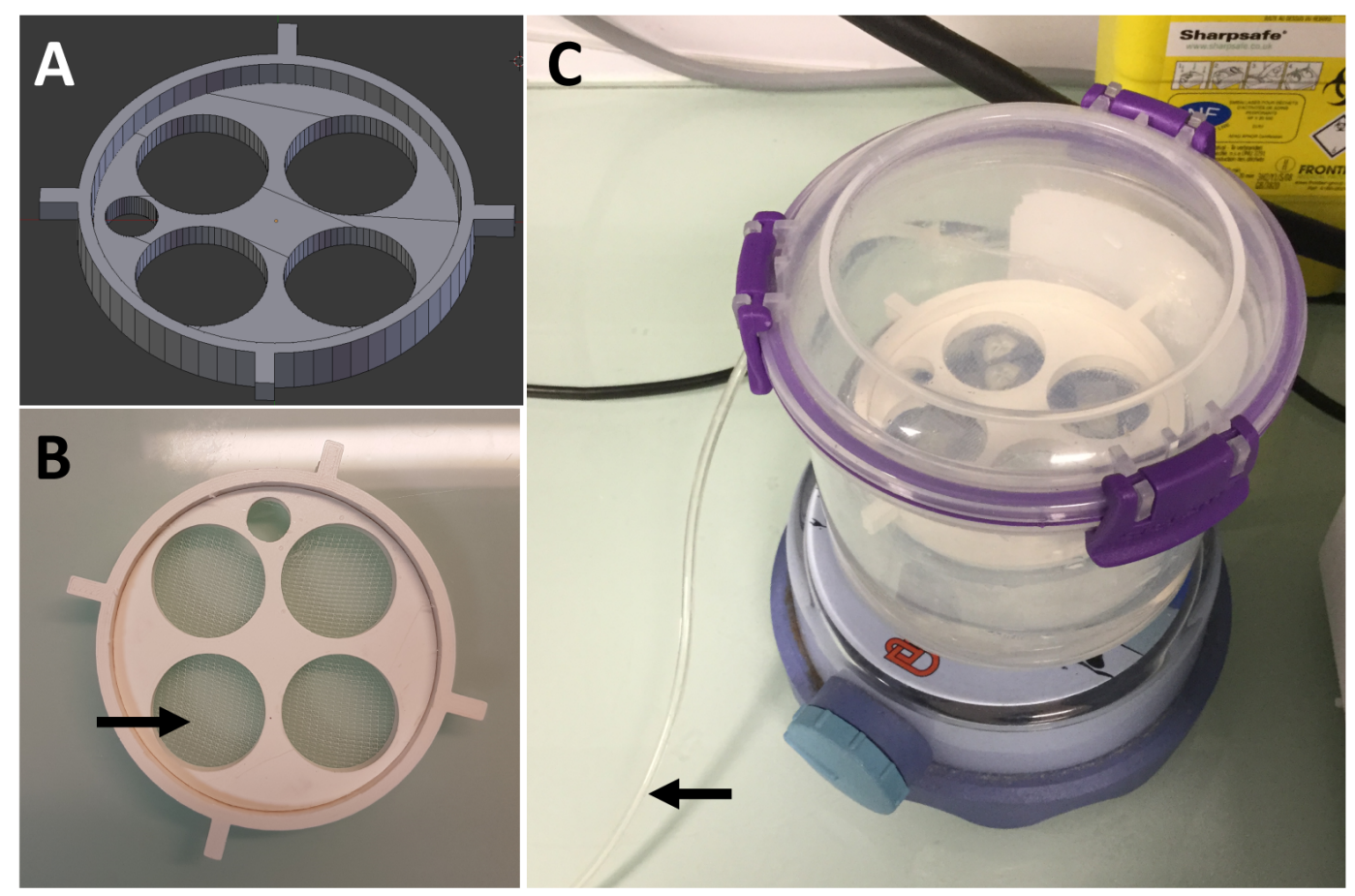

Figure 1 


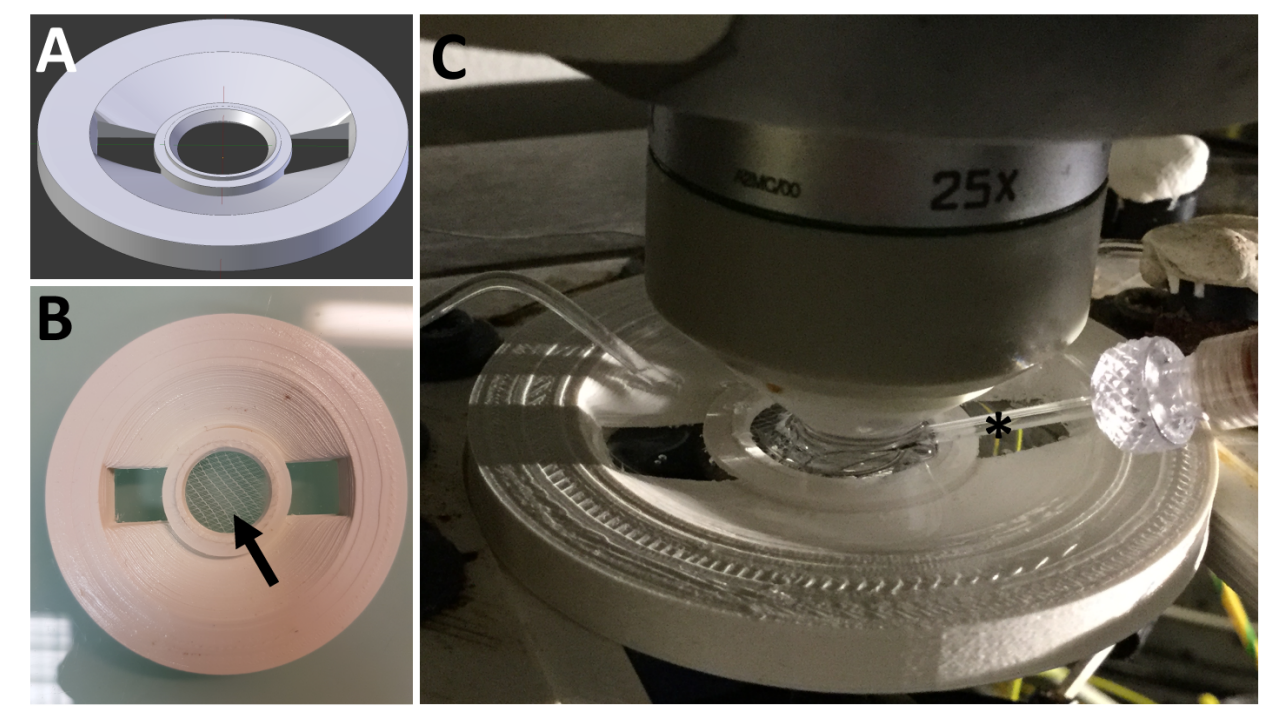

Figure 2 


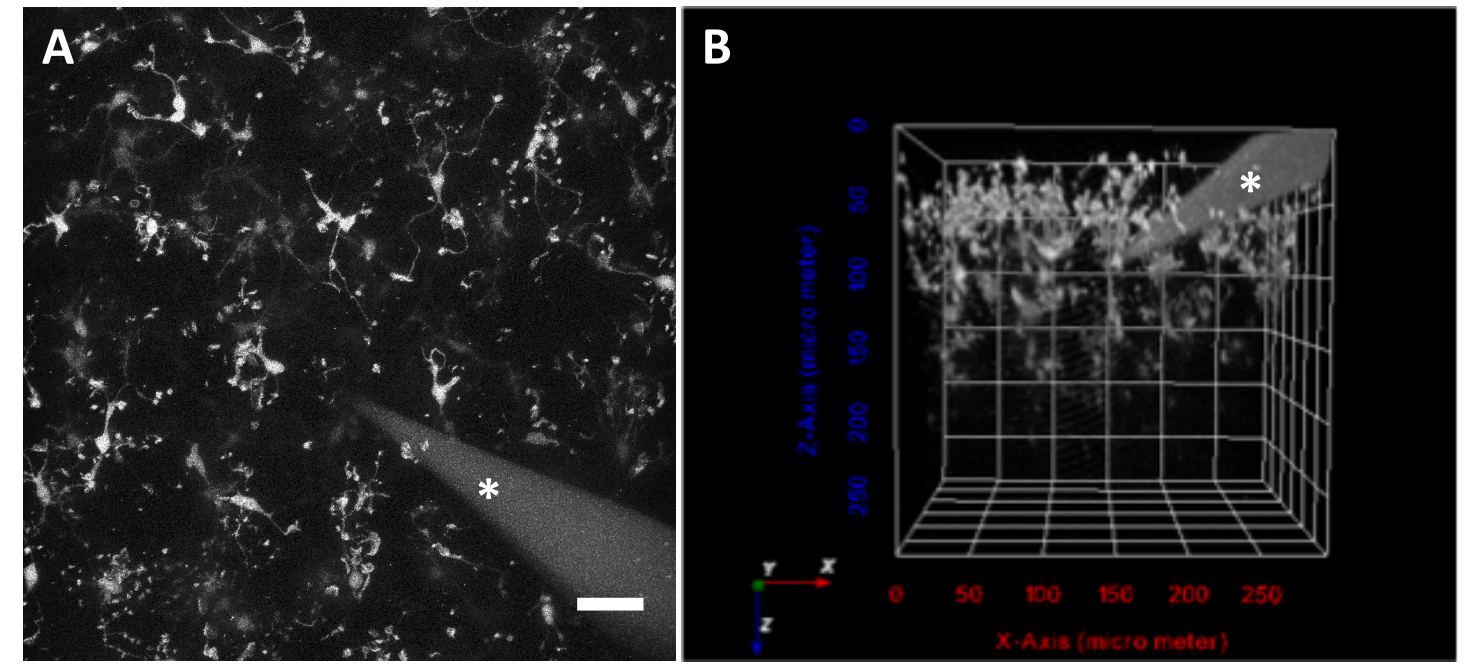

Figure 3 

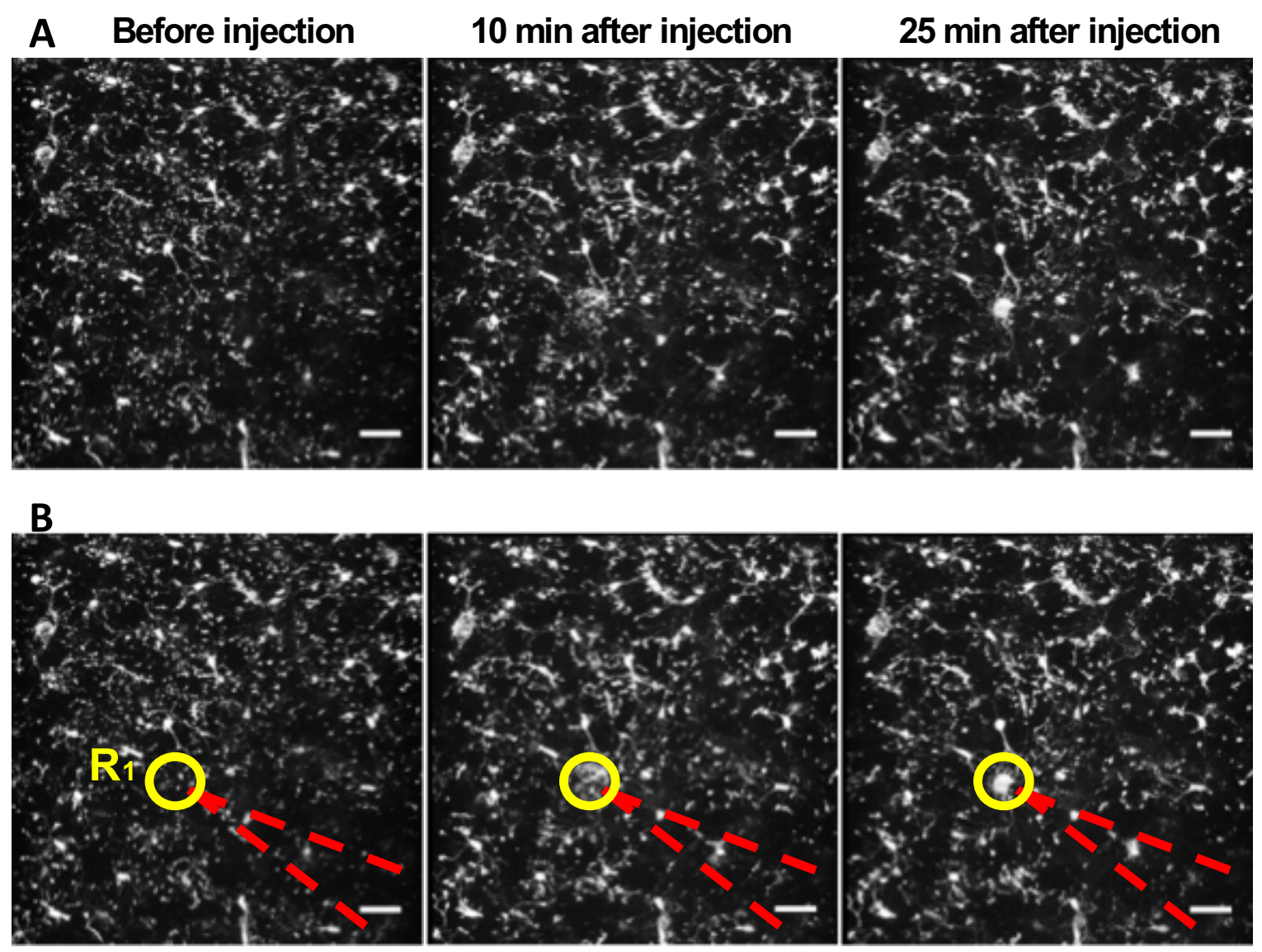

Figure 4 


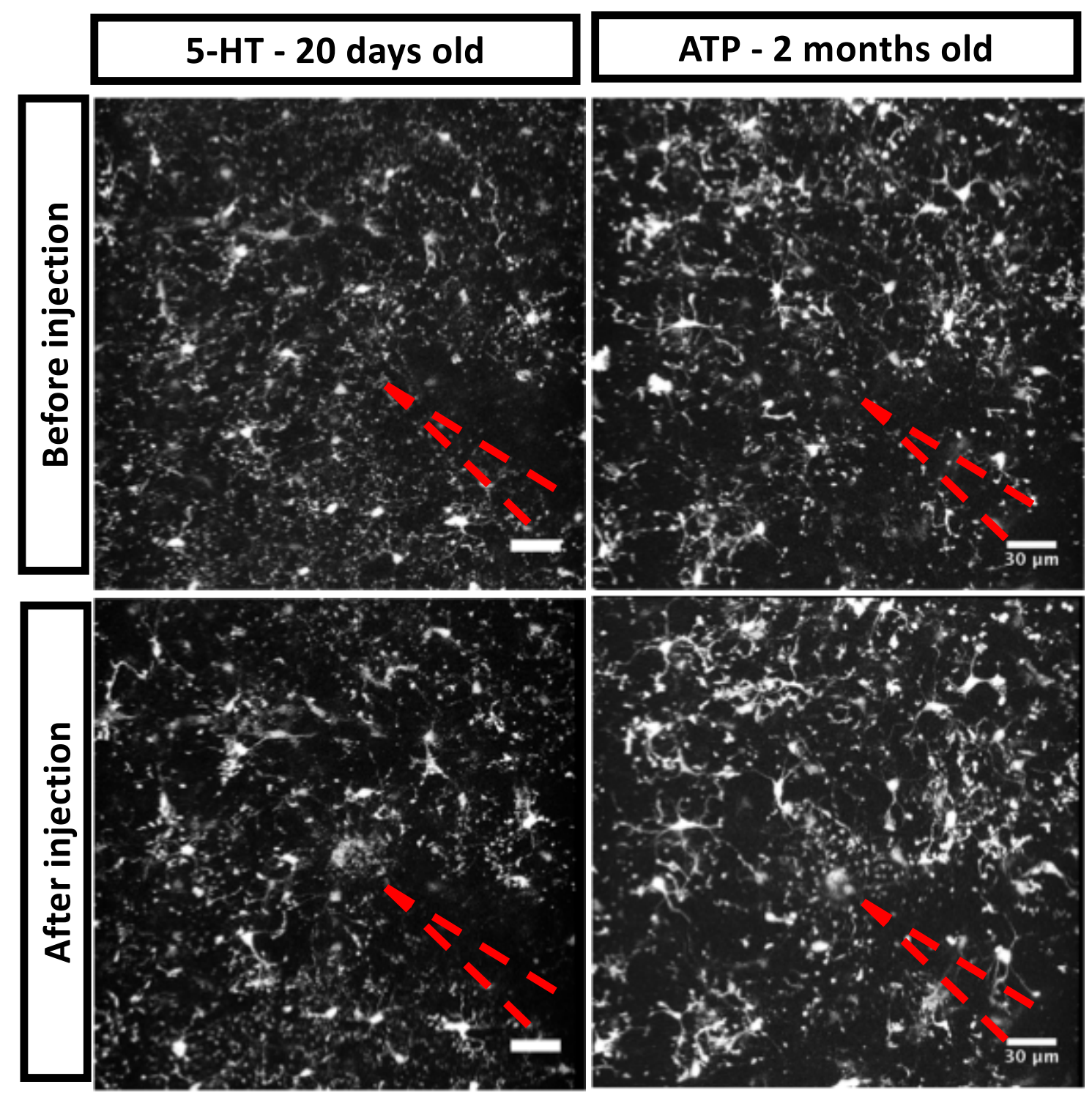

Figure 5 

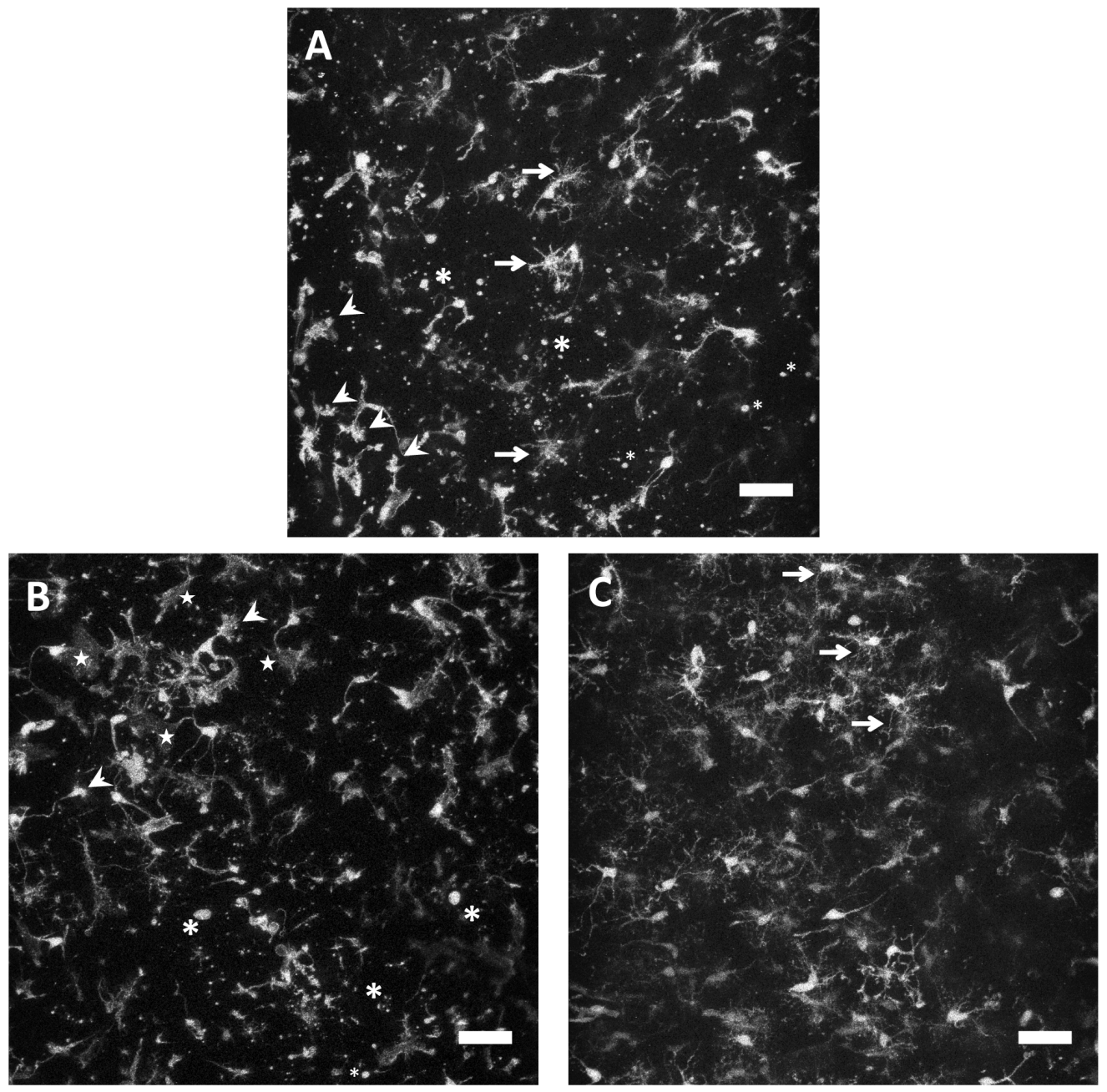

Figure 6 

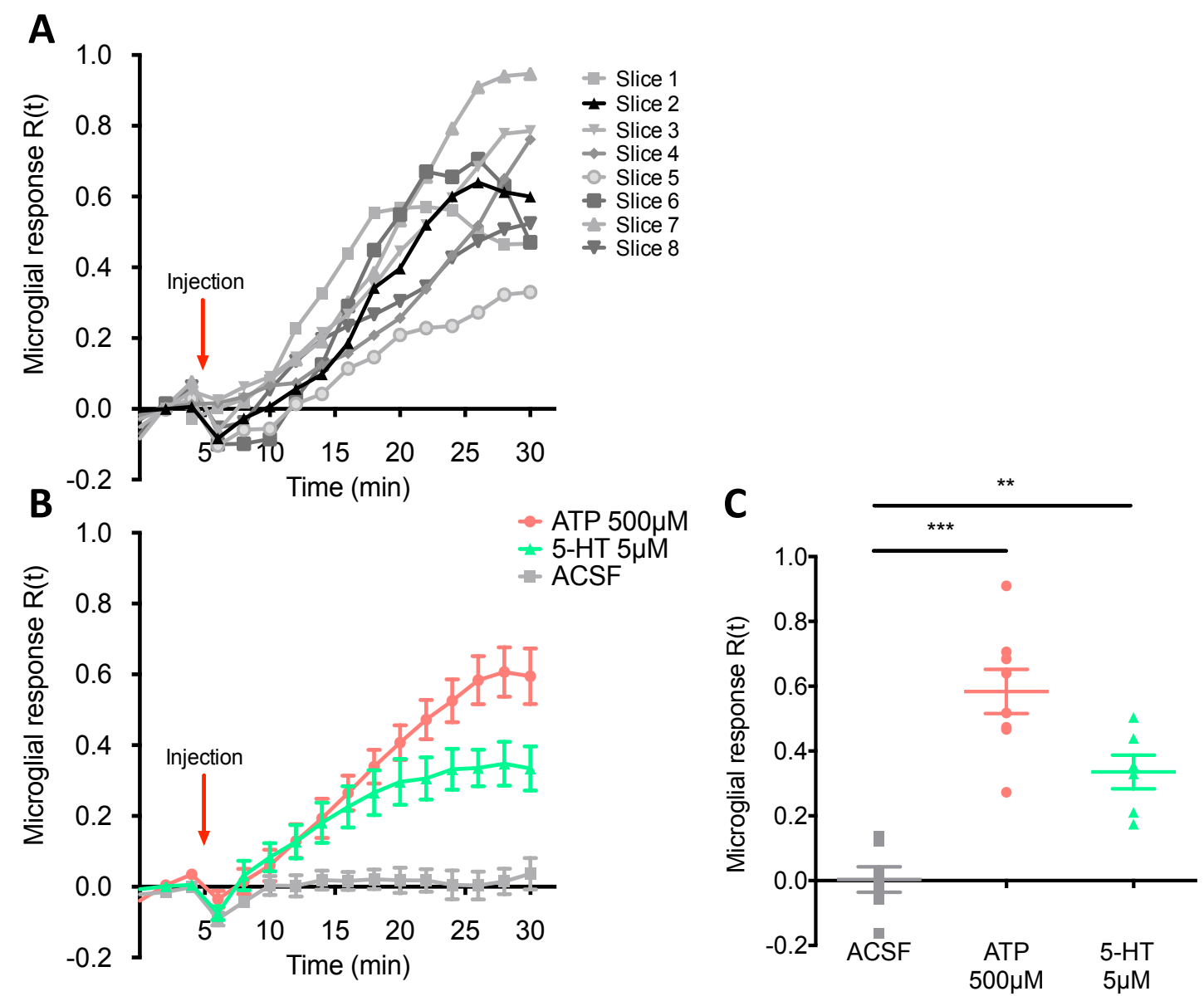

Figure 7 